\title{
Resonant Auger electron-photoion coincidence study of the fragmentation dynamics of acrylonitrile molecule
}

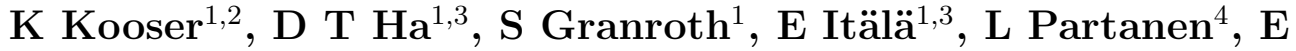 \\ Nõmmiste ${ }^{1,2}$, H Aksela ${ }^{4}$ and E Kukk ${ }^{1,5}$ \\ ${ }^{1}$ Dept. of Physics, University of Turku, FIN-20014 Turku, Finland \\ ${ }^{2}$ Institute of Physics, University of Tartu, 51014 Tartu, Estonia \\ ${ }^{3}$ Graduate School of Materials Research, Turku, Finland \\ ${ }^{4}$ Dept. of Physics, University of Oulu, Box 3000, FIN-90014 Oulu, Finland \\ ${ }^{5}$ Turku University Centre for Materials and Surfaces (MatSurf), Turku, Finland \\ E-mail: kunkoo@utu.fi
}

\begin{abstract}
Monochromatic synchrotron radiation was used to promote $K$-shell electrons of nitrogen and carbon from the cyano group $(C \equiv N)$ of gaseous acrylonitrile $\left(C_{2} H_{3}-C N\right)$ to the unoccupied antibonding $\pi_{C \equiv N}^{*}$ orbital. Photofragmentation of acrylonitrile molecules following selective resonant core excitations of carbon and nitrogen core electrons to the $\pi_{C \equiv N}^{*}$ orbital were investigated using the electron-energy-resolved photoelecton-photoion coincidence (PEPICO) technique. The fragment ion mass spectra were recorded in coincidence with the resonant Auger electrons, emitted in the decay process of the core excited states. Singly and triply deuterated samples were used for fragment identification. The results showed the initial core hole localization to be of minor importance in determining the dissociation pattern of the molecular cation. The participator and spectator Auger transitions produce entirely different fragmentation patterns and the latter indicates that complex nuclear rearrangements take place. It is suggested that the calculated kinetic energy releases are caused by the existence of metastable states, which appear with the opening of the spectator Auger channels.
\end{abstract}

PACS numbers: 33.80, 33.20, 33.15, 31.15 


\section{Introduction}

Acrylonitrile (also known as cyanoethylene, propenenitrile, and vinyl cyanide) may be regarded as a derivate of ethylene $\left(\mathrm{C}_{2} \mathrm{H}_{4}\right)$ by substituting one of the hydrogen atoms with the cyano group $(C \equiv N)$ or in other words it is olefinic nitrile containing one double bonded $C=C$ moiety together with a triple bonded $C N$ group. This molecule is an important industrial chemical: it is widely used as a monomer or comonomer for synthetic fibers, resins, plastics, elastomers and rubber. Acrylonitrile contributes heat, chemical, solvent, and weathering resistance to polymers. It is used in fumigants as well. On the other hand acrylonitrile plays an important role in astrochemistry, since this molecule and its fragments caused by cosmic radiation are present in interstellar medium. This has been a stimulus for investigations of the ion-molecule chemistry of acrylonitrile and its fragments $[1,2,3,4]$. There is a number of experimental and theoretical studies concerning the inner- and valence-shell excitations $[5,6,7,8,9,10]$ and the core-level photoemission of gaseous and adsorbed acrylonitrile $[11,12,13,14,15,16]$ as well. In the case of solid acrylonitrile the non-radiative (electronic) decay channels at the nitrogen $K$-edge have been studied by resonant Auger electron spectroscopy (RAES)[17] and for the gas phase acrylonitrile have been published the mass spectrometric investigation results of ionization and appearance energies for all detected cations [18]. Recently the kinetic energy release distribution for the metastable decay reactions of ions formed by electron impact on acrylonitrile has been reported [19]. The possible molecular and radical elimination channels in acrylonitrile photodissociation in the vacuum-ultraviolet region have been investigated by many different experimental techniques - flash photolysis-kinetic absorption spectroscopy [20], laser-induced fluorescence spectroscopy (LIF) [21], transient frequency-modulation spectroscopy [22], photofragment translational spectroscopy (PTS) [23], time-resolved Fourier transform infrared emission spectroscopy [24]. Also the theoretical calculations of reaction pathways for the unimolecular fragmentation of acrylonitrile in the ground state were thoroughly examined and it was found that the H-atom migration reactions are rapid and influence significantly the following dissociation processes [25, 26].

In the present study, the photodissociation and possible geometrical rearrangements of acrylonitrile molecule induced by the core level electron excitations are explored and discussed depending on the character of the preceding electronic relaxation channels and/or the kinetic energy of ejected Auger (or photo-)electrons. The experimental data of recently published article about the fragmentation patterns of doubly charged acrylonitrile following $C 1 s$ core ionization has been collected with the same experimental setup as the spectra of present work [27].

Next, we present the background to the processes involved. Firstly, consider the evolution of events following the interaction with soft x-rays. The subsequent description is confined to core-level photoexcitation only, as is the case of this study. The photoexcitation of a molecule promotes an electron from the core level to an initially unoccupied orbital creating a high-energy intermediate core-excited state, the lifetime of which is a few femtoseconds. The subsequent relaxation process leads to the autoionization of the excited neutral molecule and may be followed by photodissociation of the molecular cation (see Figure 1):

$$
M+h \nu \rightarrow M^{*} \rightarrow m^{+}+n+e_{\text {Auger }}^{-},
$$

where $M^{*}$ is the excited molecule and $e_{\text {Auger }}^{-}$is the resonant Auger electron with the characteristic 




Figure 1. Single photoexcitation of the neutral molecule $(M)$ to excited state $\left(M^{\star}\right)$, the following relaxation to the ionized state $\left(M^{+}\right)$or/and to the fragments $\left(m^{+}+n\right)$ depicted in the potential energy space. The symbols $I E\left(M^{+}\right)$and $A E\left(m^{+}\right)$denote the ionization energy of the precursor molecule and appearance energy of an ion fragment, respectively. Horizontal axis corresponds to the internuclear coordinate.

kinetic energy. The dissociation products in the present case are a fragment cation and a neutral fraction ( $\mathrm{m}^{+}$and $n$, respectively). In terms of energy redistribution, the above process is represented as

$$
E_{\text {int }}+E X=E_{h \nu}-E_{k i n}\left(e_{\text {Auger }}^{-}\right),
$$

where $E_{\text {int }}$ is the internal energy of the system (molecular ion or ionized and neutral fraction) and $E X$ is the adiabatic ionization energy of the neutral precursor (or appearance energy of an ion fragment). This formula implies that it is possible to extract very versatile information from the electron-energy-resolved $\left(E_{k i n}\right)$ electron-ion coincidence experiments about the formation and fragmentation dynamics of molecular cations formed by single ionization process (see, e.g., refs. $[28,29]$ and articles in ref. [30]).

We have investigated the photodissociation of acrylonitrile $\left(C_{H} H_{2}=C_{C} H-C_{N} \equiv N\right)$ molecules following resonant core excitations of nitrogen and carbon $1 s$ electrons to the molecular orbital of the localized $\pi_{C \equiv N}^{*}$ character using the electron-energy-resolved photoelectron-photoion coincidence (PEPICO) technique (the subscript letters used in the acrylonitrile formula indicate the non-equivalent carbon atoms). Also the total ion yields at the $N$ and $C 1 s$ absorption edges were recorded in order to determine the exact energy values of the corresponding excitation. In order to unravel the ambiguous pairs in the mass spectrum, also the experimental data from triply deuterated $\left(C D_{2}=C D-C \equiv N\right)$ and singly deuterated $\left(C H_{2}=C D-C \equiv N\right)$ acrylonitrile samples was recorded.

In addition, $a b$ initio molecular orbital calculations for appearance energies of dissociated fragments were carried out and the local equilibrium and the transition conformations of the acrylonitrile molecule have been calculated for some dissociation pathways. 


\section{Experimental setup}

The detailed description of the joint PEPICO setup of Oulu and Turku Universities used for the coincidence measurements is given in Reference [31]. In brief, the experimental PEPICO setup consists of a modified Scienta SES-100 electron energy analyser [32] and a homemade WileyMcLaren-type ion time-of-flight detector with a $400 \mathrm{~mm}$ long drift tube. The original CCDcamera-based detection system of the electron spectrometer was replaced by a resistive anode detector (Quantar) in order to achieve fast single electron position-sensitive response signal in nanosecond scale. The ion time-of-flight(TOF) spectrometer is equipped with a $77 \mathrm{~mm}$ active area Hamamatsu MCP detector followed by 10 concentric anode rings to enable a registration of the longitudinal and radial time-of-flight components of ions relative to the symmetry axis of the ion spectrometer tube. The ion detection electronics is based on a $1 \mathrm{GHz}$ waveform digitizer card (Signatec PDA 1000). The PEPICO measurements were performed in pulsed field mode, where the detection of electron by the electron analyzer triggers the clock for the TOF of the ions (true coincidences), within a certain time window. However, the ion TOF spectrometer detects also fragments (false coincidences or noncoincidences) which are not created from the initially ionized molecule. For extracting the coincidence and noncoincidence events the experimental setup contains also an additional pulse generator which provides artificial extraction pulses at a constant frequency. During the PEPICO experiment both - the electron and artificially triggered events, and the coincidence and noncoincidence data maps, respectively were recorded. Finally, a custom made Igor Pro software based data analysis macros [33] were used to subtract the false coincidence background from the initial coincidence map and retrieve the real coincidence data pattern. The experiment was executed at the undulator based soft X-ray beamline I411 at MAXII synchrotron radiation storage ring (Lund University, Sweden) [34]. The synchrotron radiation coming from undulator was monochromatized by a Zeiss SX-700 plane grating monochromator. The PEPICO system is operated in the pulsed extraction field mode and in the present experiment the extraction pulse voltage was $\pm 100 \mathrm{~V}$ across the sample region, with the drift tube held at $-525 \mathrm{~V}$. The entrance slit of the electron spectrometer was $1.6 \mathrm{~mm}$ and the pass energy was $200 \mathrm{eV}$, which gives a resolution $1.2 \mathrm{eV}$. In order to minimize the signal of the false coincidences the low counting rates of about 10 electrons/s have been used. The average number of ions per electron trigger was 0.8 and number of ions per artificial trigger 0.2 (including detector noise counts). The base pressure in the end-station chamber was less than $5 \times 10^{-7}$ mbar. During the experiment the chamber pressure was maintained below $5 \times 10^{-6}$ mbar. The measurements were made at room temperature. The samples of acrylonitrile and its isotopologues were purchased from Sigma-Aldrich with purity greater than $98 \%$. The further purification process was performed only through the degassing the liquid sample by multiple freeze-pump-thaw cycles before leading the sample vapor into the measurement chamber. 


\section{Results}

\subsection{Photoexcitation and resonant non-radiative decay}

The acrylonitrile molecule is planar in its electronic ground state and belongs to the $C_{S}$ point group. In the following notations the $z$ axis is chosen perpendicular to the plane $(x, y)$ of the molecule. The occupied valence configuration of the ground electronic state of acrylonitrile is given by

$$
5 a^{\prime 2} 6 a^{\prime 2} 7 a^{\prime 2} 8 a^{\prime 2} 9 a^{\prime 2} 10 a^{\prime 2} 11 a^{\prime 2} 1 a^{\prime \prime 2} 12 a^{\prime 2} 2 a^{\prime \prime 2}
$$

and the energy ordering of the unoccupied molecular orbitals is:

$$
\left(3 a^{\prime \prime}\right)^{0}\left(13 a^{\prime}\right)^{0}\left(4 a^{\prime \prime}\right)^{0}
$$

According to the earlier studies $[35,36,6,9,8]$ the orientation, localization and orbital type of these states are as follows:

the bonding MOs :

1) $5 a^{\prime}, 6 a^{\prime}, 7 a^{\prime}$ - inner valence $\sigma$ character MOs,

2) $8 a^{\prime}$ - $s$-type orbital with $C_{2 s}$ character localized in cyano group,

3) $9 a^{\prime}, 10 a^{\prime}, 11 a^{\prime}$ - correspond to $\sigma_{C-C}, \sigma_{C H_{2}}$ and $\sigma_{C \equiv N}$, respectively,

4) $1 a^{\prime \prime}$ - mixed character of $\pi_{z}(C \equiv N)$ and $\pi_{z}(C=C)$,

5) $12 a^{\prime}$ - localized $\pi_{x y}(C \equiv N)$ character,

6) $2 a^{\prime \prime}$ - delocalized conjugation of $\pi_{z}(C \equiv N)$ and $\pi_{z}(C=C)$ orbitals;

the antibonding MOs:

7) $3 a^{\prime \prime}-\pi_{z}^{*}$ orbital, delocalized along the $C_{H}=C_{C}-C_{N} \equiv N$ chain,

8) $13 a^{\prime}-\pi_{x y}^{*}$ orbital, localized on cyano $(C \equiv N)$ group

and

9) $4 a^{\prime \prime}$ - the highest $\pi_{z}^{*}$ orbital, delocalized along the $C_{H}=C_{C}-C_{N} \equiv N$ chain.

On the basis of the character of abovementioned MOs and theoretical calculations [8], it is clear, that core electron excitations to the initially unoccupied $13 a^{\prime}$ orbital can take place mostly from the $C_{N}$ and $N 1 s$ orbitals, since there is considerable overlap only between the $1 s$ wavefunctions of these atoms and the final wave function of the excited electron. It is also noteworthy to mention that only these core level transitions $\left(C_{N}(\right.$ and $\left.N) 1 s \rightarrow \pi_{x y}^{*}(C \equiv N)\right)$ have localized nature.

In the total ion yield spectra of acrylonitrile at the nitrogen and carbon $K$-edges (Figure 2 ), we observed strong resonances at $399.84 \mathrm{eV}$ and at $286.80 \mathrm{eV}$ which correspond to the transitions $N 1 s \rightarrow \pi_{x y}^{*}(C \equiv N)$ and $C_{N} 1 s \rightarrow \pi_{x y}^{*}(C \equiv N)$, respectively. The beamline monochromator resolution was $0.3 \mathrm{eV}$ and $0.4 \mathrm{eV}$ for incident radiation at corresponding $C$ and $N K$-edges.

Resonant Auger decay spectra of these two excitations are shown in Figure 3 in comparison with a direct photoionization spectrum taken at ionization energy of $60 \mathrm{eV}$ and with $25 \mathrm{meV}$ photon energy resolution. Both resonant decay spectra possess a similar structure with two clearly separated maxima. The structure in the binding energy range between 11 and $15 \mathrm{eV}$ corresponds to 


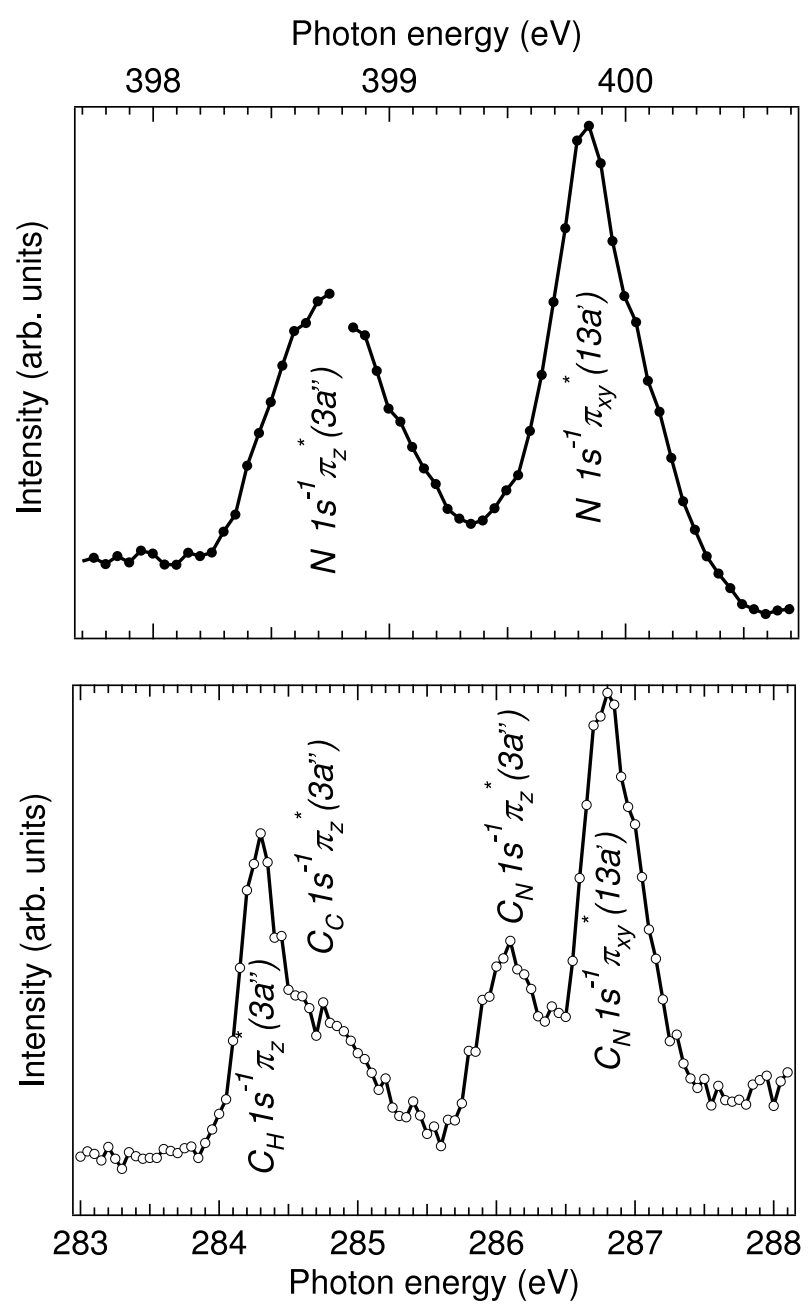

Figure 2. Upper panel: total ion yield of acrylonitrile at nitrogen $K$-edge. Lower panel:total ion yield of acrylonitrile at carbon $K$-edge. The labels indicate the corresponding excited states.

the participator transitions, where the photoexcited electron is directly involved in the deexcitation process leading to the single-hole final states as in the direct ionization from $11 a^{\prime}$ and $12 a^{\prime}$ orbitals. The high binding energy side of the deexcitation spectra has a broad structure from 16 up to $30 \mathrm{eV}$ with the maximum at around $21 \mathrm{eV}$. In this region the nonresonant electron energy spectrum has only weak inner valence and electron-correlation satellite ionization states. This spectral region can be attributed to the different spectator decay channels, which are in more detail described in Ref.[17]. This decay channel leads to a two hole-one electron final state where the excited electron acts as a localized spectator and screens the final two hole states.

\subsection{Photofragmentation pathways}

Figures 4 and 5 depict the PEPICO maps with corresponding integrated ion TOF and electron energy spectrum recorded at the $N$ and $C_{N} 1 s \rightarrow \pi_{x y}^{*}(C \equiv N)$ resonant excitation energy, respectively. The spread of electron kinetic energy window, which is determined by the pass energy of electron spectrometer, was chosen to cover the range of corresponding resonant $K V V$ Auger structures. The coincidence measurement setup and extraction of real coincidence dissociation 


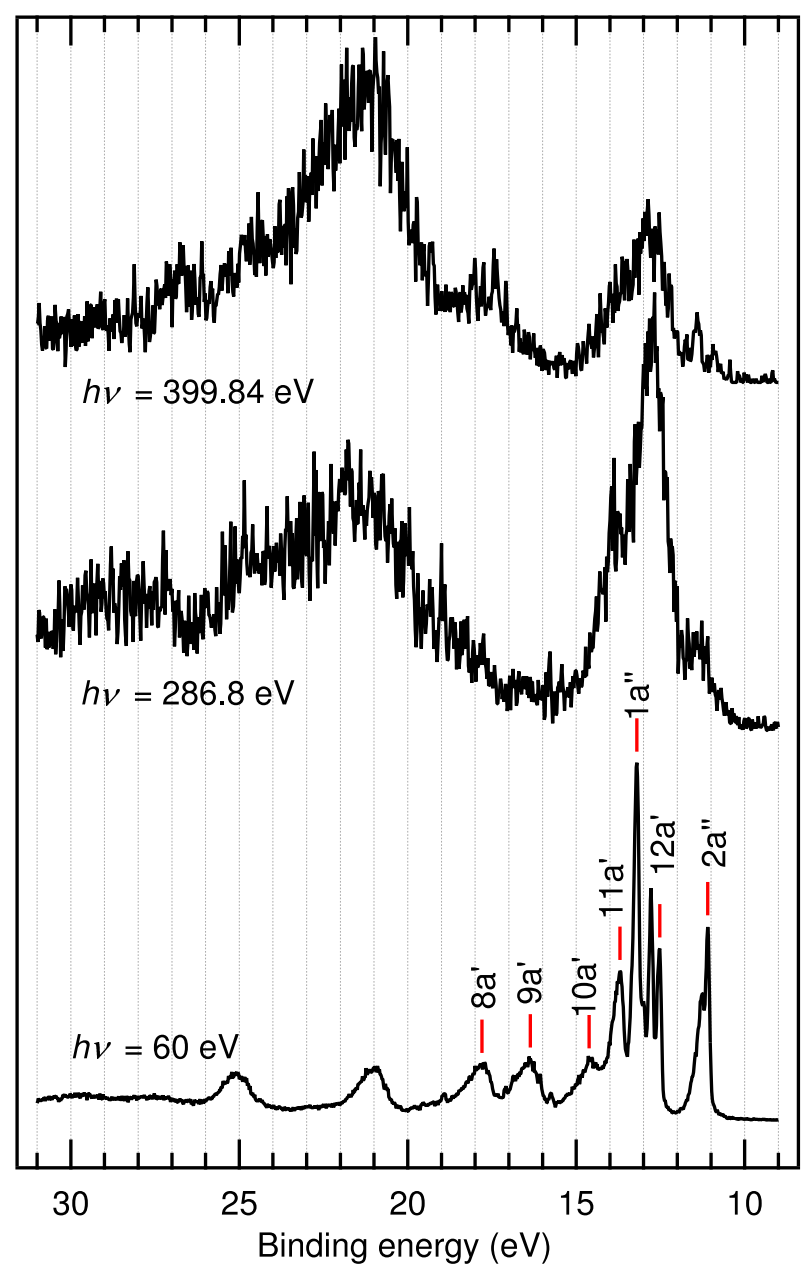

Figure 3. Electron spectra of gaseous acrylonitrile measured at marked excitation energies. The labels above the lower spectrum indicate the ionization energies of acrylonitrile valence molecular orbitals. Two upper spectra correspond to the resonant Auger decay after $N 1 s \rightarrow \pi_{C \equiv N}^{*}$ and $C_{N} 1 s \rightarrow \pi_{C \equiv N}^{*}$ excitations, respectively.

pattern has been expounded in the experimental setup section.

The ion TOF spectrum shows a large variety of different dissociation fragments including the precursor molecular ion $\mathrm{C}_{3} \mathrm{H}_{3} N^{+}$. Unfortunately the equal mass fragment pairs like $\left(\mathrm{CH}_{2}^{+}, \mathrm{N}^{+}\right),\left(\mathrm{CH}_{3}^{+}, \mathrm{NH}^{+}\right),\left(\mathrm{C}_{2} \mathrm{H}_{2}^{+}, \mathrm{CN}^{+}\right),\left(\mathrm{C}_{2} \mathrm{H}_{3}^{+}, \mathrm{CNH}^{+}\right),\left(\mathrm{CNH}_{2}^{+}, \mathrm{N}_{2}^{+}\right),\left(\mathrm{C}_{3} \mathrm{H}_{2}^{+}, \mathrm{C}_{2} \mathrm{~N}^{+}\right)$and $\left(\mathrm{C}_{3} \mathrm{H}_{3}^{+}, \mathrm{C}_{2} \mathrm{NH}^{+}\right)$are not discernible in time-of-flight ion spectrum. In order to identify the ambiguous fragments from the coincidence ion TOF, we performed also the PEPICO measurements of the deuterated acrylonitrile which has different fragment pairing. Using the curve fitting analysis [33], we determined the intensities of all TOF peaks for both samples and composed the systems of linear equations, where the variables were the abundancies of the possible fragments with the same mass-to-charge ratio. The last step was to use least-squares minimization to achieve the best agreement between the calculated abundancies and the curve-fitted TOF intensities. The list of dissociation products according to this analysis is shown in Table 1 . The light $H^{+}$and $D^{+}$ fragments are not added to this table due to the unreliability of quantitative intensity information, which is caused by the low detection efficiency of these fast fragments. 


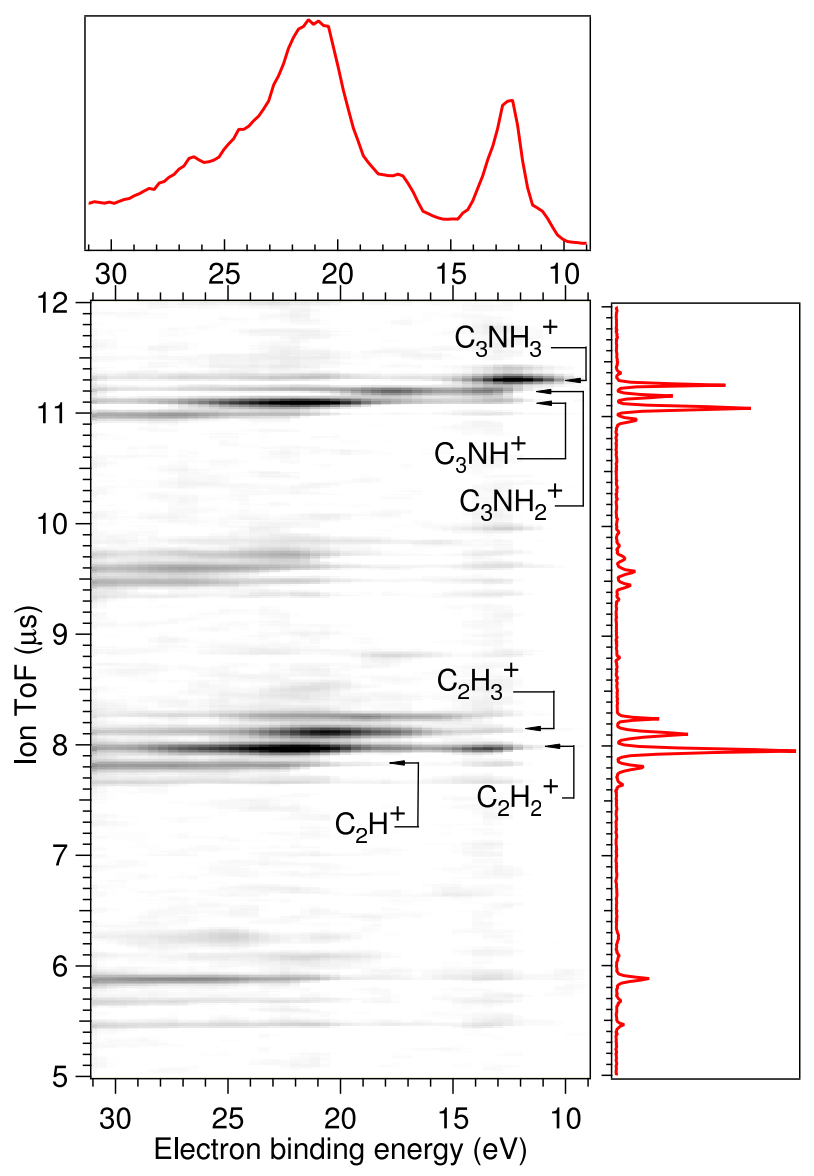

Figure 4. Electron-ion coincidence (PEPICO) map of acrylonitrile from the Auger decay of the $N 1 s^{-1} \pi_{x y}^{*}$ resonance at $h \nu=399.84 \mathrm{eV}$. The electron energy spectrum (top panel) and the ion TOF spectrum (right-hand panel) are both integrated from the data of the PEPICO map (main panel). The labels with arrows denote the main dissociation fragments.

The main fragments of acrylonitrile following the $N 1 s \rightarrow \pi_{C \equiv N}^{*}$, and $C_{N} 1 s \rightarrow \pi_{C \equiv N}^{*}$ excitations can be divided into the heavy $\mathrm{C}_{3} \mathrm{NH}_{n}^{+}$and the light $\mathrm{C}_{2} H_{n}^{+}$groups, where $n=1-3$. The relative weights of these fragmentation groups constitute approximately $77 \%$ and $66 \%$ of the recorded fragmentation pattern of acrylonitrile following the corresponding resonant excitations. The comparison of relative intensity weights of fragment pairs $\left(\mathrm{C}_{2} \mathrm{H}^{+}, \mathrm{CNH}_{2}^{+}\right),\left(\mathrm{C}_{2} \mathrm{H}_{2}^{+}, \mathrm{HCN}^{+}\right)$ and $\left(\mathrm{C}_{2} \mathrm{H}_{3}^{+}, \mathrm{CN}^{+}\right)$also implies that the singly ionized fragment cations are formed more probably from the moieties containing vinyl $\left(\mathrm{CH}_{2}=\mathrm{CH}-\right)$ than from the cyano $(-\mathrm{C} \equiv N)$ group.

\subsection{Electronic relaxation channel dependent fragmentation}

The main panels of Figures 4 and 5 display the fragment and molecular ion intensities depending on the energy of electrons. These coincidence fragmentation patterns also allow to study, how the fragmentation pathways depend on the atomic site of the core electron excitation, as the initially unoccupied $\pi_{x y}^{*}(C \equiv N)$ is strongly localized in the neighborhood of the cyano group.

Examination of both PEPICO patterns indicates no major variances between their dissociation products (see also Table 1). This leads to the conclusion that, even in the present case where the initial perturbation has a well localized nature, the atomic site of initial core excitation has no 




Figure 5. Electron-ion coincidence (PEPICO) map of acrylonitrile from the Auger decay of the $C 1 s^{-1} \pi_{x y}^{*}$ resonance at $h \nu=286.8 \mathrm{eV}$. The electron energy spectrum (top panel) and the ion TOF spectrum (right-hand panel) are both integrated from the data of the PEPICO map (main panel).

major influence to the following fragmentation process. In other words, the $C_{N} 1 s$ and $N 1 s$ core level excitations lead to the final electronic states with very similar populations (see the two upper decay spectra in Figure 3) as a starting point of the dissociation.

In order to explore in detail the energy dependence of the creation of fragment cations, the data of the PEPICO map in Figure 4 have been processed to the form of the coincidence ion yields (CIYs), which represent the ion intensities as a function of the coincident electron's binding energy (or as a function of the total available excess internal energy of the parent molecular cation). Also the experimental appearance energies for the main fragments have been estimated by linear interpolation of the coincidence ion yield curves. In Figure 6 are shown the CIYs of the acrylonitrile precursor ion and five main fragment cations, following the $N 1 s^{-1} \pi_{x y}^{*}(C \equiv N)$ excitation and in coincidence with the corresponding resonant $K V V$ Auger electrons. The ionization of the parent molecule starts at $10 \mathrm{eV}$ binding energy and has the peak intensity at $12.4 \mathrm{eV}$, which coincides with the maximum of the participator structure of the electron spectrum and is in resonance with the direct ionization final state $\left(12 a^{\prime}\right)^{-1}$. The participator transitions lead also to the fragmentation of the acrylonitrile molecule. The pattern of the $\mathrm{C}_{3} \mathrm{H}_{2} \mathrm{~N}^{+}$ion appears at around $12 \mathrm{eV}$ and concurrent loss of a neutral $\mathrm{H}$ atom starts to take place. The intensity distribution of the $\mathrm{C}_{3} \mathrm{H}_{2} \mathrm{~N}^{+}$ion extends up to $23 \mathrm{eV}$ in the binding energy scale and has two maxima at 13.3 and $17.4 \mathrm{eV}$. The first one 
Table 1. Photofragmentation products of the $C_{3} H_{3} N$ and $C_{3} D_{3} N$ molecules following the $C 1 s \rightarrow \pi^{*}$ and $N 1 s \rightarrow \pi^{*}$ core excitations. $H$ or $D$ are commonly denoted by $X$. Masses of the deuterated fragments are given in brackets. Abundancies are given in percent together with error bars in brackets.

\begin{tabular}{|c|c|c|c|}
\hline & & \multicolumn{2}{|c|}{ Abundancy } \\
\hline Fragment & Mass (a.m.u.) & $C 1 s \rightarrow \pi^{*}$ & $N 1 s \rightarrow \pi^{*}$ \\
\hline$C X^{+}$ & $13(14)$ & $1.2(0.1)$ & $0.9(0.1)$ \\
$N^{+}$ & 14 & $0.1(0.2)$ & $0.2(0.3)$ \\
$C X_{2}^{+}$ & $14(16)$ & $1.9(0.4)$ & $1.3(0.4)$ \\
$N X^{+}$ & $15(16)$ & $1.3(0.1)$ & $0.8(0.1)$ \\
$C X_{3}^{+}$ & $15(18)$ & $0.0(0.1)$ & $0.0(0.1)$ \\
$N X_{2}^{+}$ & $16(18)$ & $1.1(0.1)$ & $0.9(0.1)$ \\
\hline$C_{2} X^{+}$ & $25(26)$ & $7.4(0.1)$ & $5.2(0.1)$ \\
$C_{2} X_{2}^{+}$ & $26(28)$ & $23.7(0.7)$ & $28.5(0.8)$ \\
$C N^{+}$ & 26 & $2.2(0.5)$ & $0.0(0.5)$ \\
$C_{2} X_{3}^{+}$ & $27(30)$ & $7.1(0.3)$ & $10.9(0.4)$ \\
$C N X^{+}$ & $27(28)$ & $2.0(0.5)$ & $2.2(0.6)$ \\
$C N X_{2}^{+}$ & $28(30)$ & $2.6(0.1)$ & $3.3(0.1)$ \\
\hline$C_{3} X^{+}$ & $37(38)$ & $4.0(0.1)$ & $2.4(0.1)$ \\
$C_{3} X_{2}^{+}$ & $38(40)$ & $1.8(0.5)$ & $1.1(0.5)$ \\
$C_{3} X_{3}^{+}$ & $39(42)$ & $0.0(0.1)$ & $0.0(0.1)$ \\
$C_{2} N^{+}$ & 38 & $3.9(0.4)$ & $2.3(0.3)$ \\
$C_{2} N X^{+}$ & $39(40)$ & $2.0(0.2)$ & $2.1(0.2)$ \\
$C_{2} N X_{2}^{+}$ & $40(42)$ & $0.3(0.1)$ & $0.7(0.1)$ \\
$C_{2} N X_{3}^{+}$ & $41(44)$ & $0.3(0.1)$ & $0.1(0.1)$ \\
\hline$C_{3} N X^{+}$ & $51(52)$ & $12.6(0.3)$ & $18.5(0.3)$ \\
$C_{3} N X_{2}^{+}$ & $52(54)$ & $5.7(0.2)$ & $5.8(0.2)$ \\
$C_{3} N X_{3}^{+}$ & $53(56)$ & $9.8(0.2)$ & $7.9(0.2)$ \\
\hline$C^{+}$ & 12 & $1.8(0.1)$ & $1.0(0.1)$ \\
$C_{2}^{+}$ & 24 & $1.2(0.1)$ & $0.8(0.1)$ \\
$C_{3}^{+}$ & 36 & $0.5(0.1)$ & $0.0(0.1)$ \\
$C_{3} N^{+}$ & 50 & $3.9(0.1)$ & $3.0(0.1)$ \\
\hline & & &
\end{tabular}

coincides with the state $\left(11 a^{\prime}\right)^{-1}$ and the second occurs in the low binding energy side of spectator Auger region. The $C_{3} H N^{+}$fragment appears at $11.5 \mathrm{eV}$ with very slow intensity increase up to $18.5 \mathrm{eV}$ binding energy and then the intensity of this fragment starts to follow the shape of the spectator structure of the electron spectrum. The co-product of this dissociation channel is molecular $\mathrm{H}_{2}$ which is energetically more favored compared to the release of two atomic $H$.

In addition to the production of heavy fragments at the energy region of the participator transitions, the rise of lighter $\mathrm{C}_{2} \mathrm{H}_{2}^{+}$ion pattern and the loss of neutral hydrogen cyanide $(H C N)$ molecule starts. The appearance threshold of this fragment is at $11.8 \mathrm{eV}$ and the first intensity maximum is located at $13.3 \mathrm{eV}$. It can be seen from Figure 6 that the elimination channels of 


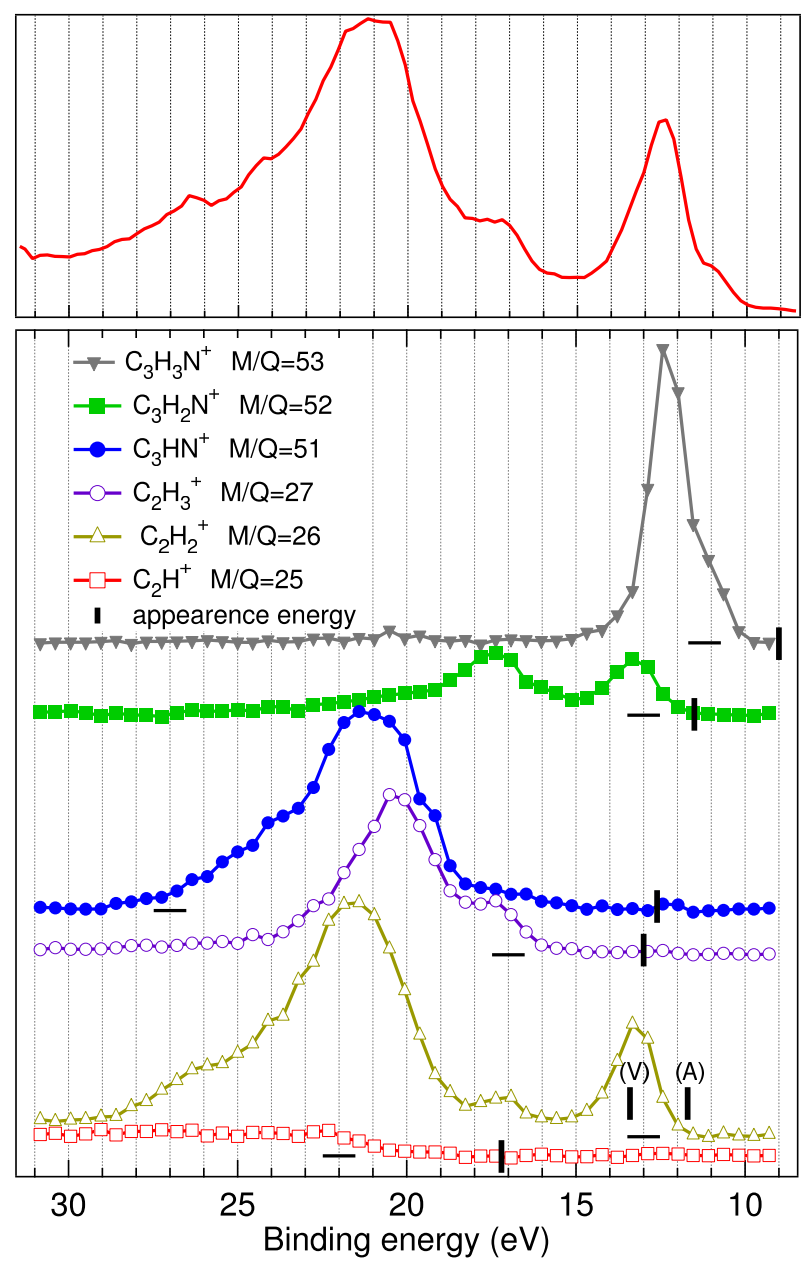

Figure 6. Coincidence ion yields (CIY) of acrylonitrile main fragments following the Auger decay of the $N 1 s^{-1} \pi^{*}$ resonance at $h \nu=399.84 \mathrm{eV}$ and the electron energy spectrum on top panel. The calculated appearance energy values for each CIY curve are given by vertical (black) bars. Labeled appearance energy bars (A), (V) correspond to the acetylene $(H-C \equiv C-H)$ and vinylidene (: $\mathrm{C}=\mathrm{CH}_{2}$ ) isomeric configurations of $\mathrm{C}_{2} \mathrm{H}_{2}^{+}$cation, respectively. The horizontal bars denote the zero level of signal for each CIY curve.

the $H$ atom and the $H C N$ molecule are competing at the high binding energy shoulder of the participator peak in the electron spectrum. The creation of $H$ can be explained by simple rupture of the bond between hydrogen and carbon $\left(C_{H}\right.$ or $\left.C_{C}\right)$ atoms if the molecular ion has got enough excess energy for this process. The $H C N$ elimination can be explained as follows: when the core electron is promoted to the $\pi_{C \equiv N}$ orbital, the hybridization of the $C_{N}$ atom changes from $s p$ to $s p^{2}$ and thus gives rise to the change of geometry of the acrylonitrile molecule [37]. Subsequently, the bond between $H$ and $C_{C}$ (or $C_{H}$ ) atoms weakens and the hydrogen atom starts to form partial bond(s) with the $C_{N}$ (and $N$ ) atom(s). Finally, the bond between the migrating $H$ atom and vinyl group $\left(\mathrm{C}_{2} \mathrm{H}_{2}\right)$ breaks and the $H C N$ molecule separates from the parent acrylonitrile molecular ion.

In the binding energy range corresponding to the spectator transition, three strong fragments are observable: $\mathrm{C}_{3} \mathrm{HN}^{+}, \mathrm{C}_{2} \mathrm{H}_{2}^{+}$and $\mathrm{C}_{2} \mathrm{H}_{3}^{+}$. The co-product of the latter is the neutral $\mathrm{CN}$ radical caused by the simple bond cleavage between vinyl and cyano group. 


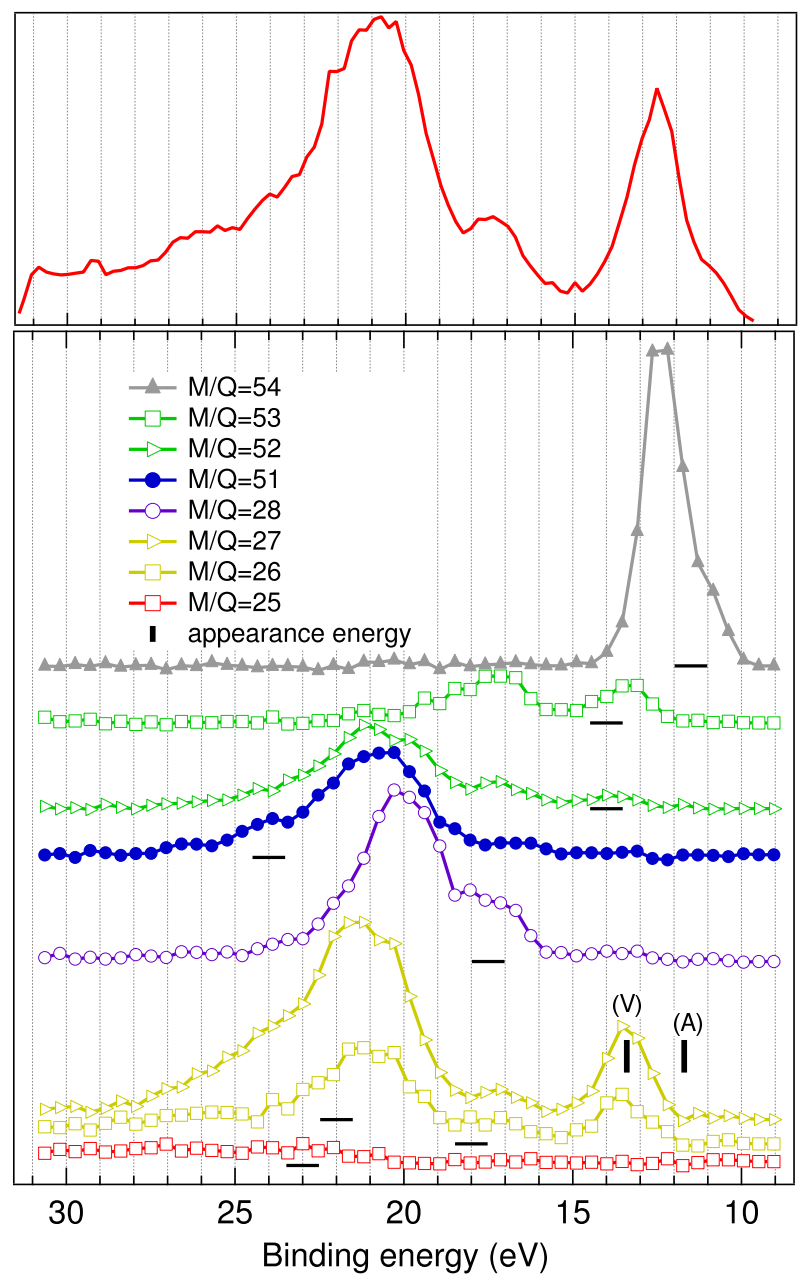

Figure 7. Coincidence ion yields (CIY) of acrylonitrile-2-d $\left(\mathrm{CH}_{2}=\mathrm{CD}-\mathrm{CN}\right)$ main fragments following the Auger decay of the $N 1 s^{-1} \pi^{*}$ resonance at $h \nu=399.84 \mathrm{eV}$ and the electron energy spectrum on top panel. The calculated appearance energy values for each CIY curve are given by vertical (black) bars. Labeled appearance energy bars $(\mathrm{A}),(\mathrm{V})$ correspond to the acetylene $(\mathrm{H}-\mathrm{C} \equiv \mathrm{C}-\mathrm{H})$ and vinylidene (: $\mathrm{C}=\mathrm{CH}_{2}$ ) isomeric configurations of $\mathrm{C}_{2} \mathrm{H}_{2}^{+}$cation, respectively. The horizontal bars denote the zero level of signal for each CIY curve.

\section{Nuclear rearrangements caused by the core level excitations and metastability of fragments}

This section will discuss the acrylonitrile fragmentation preceded by the cyano group core level excitations, concerning the possible geometrical changes during the evolution of these processes. In addition, ab initio molecular orbital calculations for appearance energies of dissociated fragments, carried out by the unrestricted Hartree-Fock (UHF) theory with the split-valence 6-311(dp) basis set [38] containing polarization functions for all atoms will be reported. Computational studies presented in this work were executed with GAMESS (The General Atomic and Molecular Electronic Structure System) program [39]. The theoretical appearance energies were estimated by substracting the total energy of the neutral parent compound from the sum of total energies of its isolated fragments. These fragments are the isomers corresponding to the conformations which have the (globally) lowest energy. The careful exploration and analysis of PEPICO maps together 
with calculations shows the relevance of geometrical readjustment to the coincidence dissociation pathways.

\subsection{Molecular dissociation pathway: $\left(\mathrm{C}_{2} \mathrm{H}_{2}^{+}, \mathrm{HCN}\right)$}

Let us turn back to Figure 6, where every CIY is presented together with the corresponding ionization or appearence energy (AE) bar. The overall agreement between experiment and calculation is excellent, except in the case of the strongest fragment $\left(\mathrm{C}_{2} \mathrm{H}_{2}^{+}\right)$, for which we calculated AEs for two possible configuration - acetylene $(H-C \equiv C-H)$ and vinylidene (: $\mathrm{C}=\mathrm{CH}_{2}$ ). It has been observed earlier that the acetylene molecular ion induced by the ionization of carbon $1 s$ electron often rearranges from the acetylene configuration to the vinylidene form prior to the dissociation in a time scale estimated to be less than $60 \mathrm{fs}$ [43]. According to the calculations [37] the periods of the $C-H$ vibrational stretching modes in acrylonitrile are comparable to the intrinsic lifetime of the core excited molecule (5-10 fs). The non-zero probability for a hydrogen atom migration may cause the complete geometrical rearrangement of excited molecule during the processes following the initial excitation.

In order to ascertain the possible geometrical configurations of $\mathrm{C}_{2} \mathrm{H}_{2}^{+}$, we performed the coincidence measurements also with singly deuterated acrylonitrile $\left(\mathrm{CH}_{2}=\mathrm{CD}-\mathrm{CN}\right.$ or acrylonitrile-2-d). The curves of CIYs of main fragments and parent ion are depicted in Figure 7. The analysis of experimental data (which method is already given in subsection 3.2) shows that the abundancy ratios of fragment pairs $\left\{\left(C_{3} H D N^{+}\right)_{M / Q=53} /\left(C_{3} H_{2} N^{+}\right)_{M / Q=52}\right\}$ and $\left\{\left(C_{2} H D^{+}\right)_{M / Q=27} /\left(C_{2} H_{2}^{+}\right)_{M / Q=26}\right\}$ approach the ratio of $2: 1$, which can be deduced by calculating the number of the 2-combinations of the 3-element set $(H, H, D)$. Secondly, the experimental appearence energies of fragments $M / Q=26$ and $M / Q=27$ are equal. The last circumstance indicates that the geometrical structure of the precursor molecular ion has undergone significant rearrangement process before the dissociation to these two fragments.

Our calculations confirm clearly this interpretation. Two possible dissociation pathways with minima and corresponding characteristic transition states are traced in Figure 8. The upper pathway conforms to the fragmentation channel, which leads to the creation of $\mathrm{C}_{2} \mathrm{H}_{2}^{+}$and lower corresponds to $\mathrm{C}_{2} \mathrm{HD}^{+}$fragment product.

It is quite obvious that the hydrogen bonded with $C_{C}$ carbon would most probably attach itself to $\mathrm{CN}$ part in the $\mathrm{C}_{2} \mathrm{H}_{2}^{+}$and $\mathrm{HCN}$ dissociation process (Figure 8a). However, the strong CIY corresponding to $\mathrm{M} / \mathrm{Q}=27$ in Figure 7 together with the theoretical appearance energy indicate that the above mentioned dissociation channel is not the only possibility. This implies that the parent compound should go through an isomerization process before the fragmentation (Figure $8 \mathrm{~b}$ ). The calculated appearance energy matching to the experimental appearance threshold of $\mathrm{M} / \mathrm{Q}=27$ was acquired by substracting the total energy of the neutral parent molecule from the sum of total energies of geometry-optimized singly charged acetylene $\left(H C C D^{+}\right)$, which is the global minimum of all the possible $\mathrm{C}_{2} \mathrm{H}_{2}^{+}$conformations) and neutral $H C N$. Accordingly, using modelling result of $\mathrm{HCCH}^{+}$compound to attempt to describe the singly deuterated one $\left(\mathrm{HCCD}^{+}\right)$is reasonable, since the effect of deuterating the sample is assumingly minimal [4] and of course negligible in sense of the Hartree-Fock theory. The isomerization process can be qualitatively modelled by examining the potential hypersurface of the molecule. The hypersurface is the function of $\left(3^{*} 7-6=\right)$ 
(a)

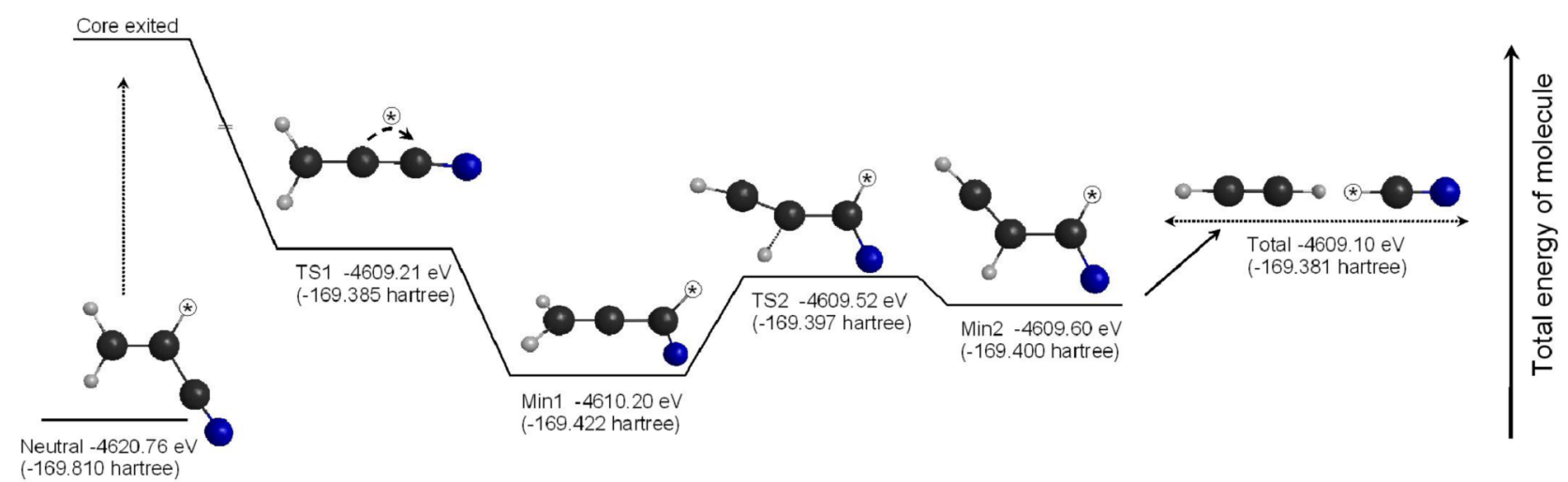

(b)

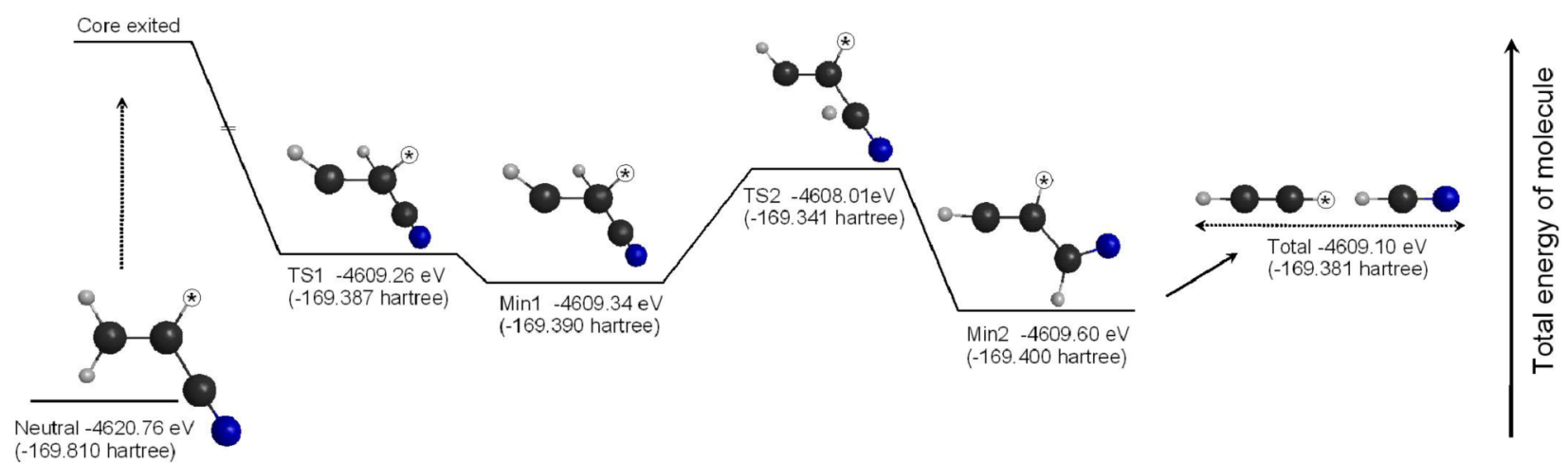

Figure 8. Two possible photofragmentation and isomerization pathways of the acrylonitrile parent ion leading to the creation of the $\mathrm{C}_{2} \mathrm{H}_{2}^{+}$ion and the loss of $\mathrm{HCN}$ molecule. The minima (Min) and corresponding characteristic transition states (TS) are given together with their total energy values in electronvolts and hartrees. The hydrogen atom (replaced with $D$ in singly deuterated sample) initially located at central carbon atom $\left(C_{C}\right)$ is denoted with asterisk $(\star)$

15 degrees of freedom representing nuclear coordinates of the atoms in the molecule. Here, our main interest lies in the local equilibrium and the transition conformations of the parent ion and also the potential barriers between the stationary points. The equilibrium conformations were located by quasi-Newton algorithm [40] and the transition structures were found by following the minimum energy path on the potential surface of the parent ion. The intermediate structures were also verified by a vibrational frequencies analysis - the vibrational motion associated with the negative frequency is the one oscillating back and forth between the initial and the final equilibrium state. Figure 8 illustrates two of the possible internal rearrangements of the dissociating acrylonitrile ion, which lead to the same final state, but the hydrogen atom initially located at central carbon atom $\left(C_{C}\right)$ ending up in different fragments. 


\subsection{Kinetic energy release of fragments depending on the electronic state}

The kinetic energy release (KER) during the dissociation results in an additional velocity spread of the fragment ions and this velocity change is reflected in the broadening of the TOF peak of fragment. The coincidence measurements permit to examine the KERs of different cations depending on the excess internal energy of parent ion. In the present case we have determined the FWHMs of TOF peaks for the $\mathrm{C}_{3} \mathrm{H}_{2} \mathrm{~N}^{+}$and $\mathrm{C}_{2} \mathrm{H}_{2}^{+}$fragments; both of them were detected in participator and also in spectator range of the electron spectrum. We used the pseudo-Voigt function for curve fitting [33] of the experimental data, since the shapes of these TOF peaks resemble more a Gaussian than flat-topped (or dish-shaped) distributions that arise at high KER values. This curve fitting approximation gives one representative value of the KERs, possibly combining several channels each with a different KER [41]. The KERs were obtained by comparing the experimental widths of peaks with Monte-Carlo simulations of the ion TOF spectra using the values of experimental extraction and acceleration voltages and the corresponding dimensions of the ion-TOF spectrometer's regions. The results of KER values of these two fragments are presented in Table 2. The simulation also shows that under the present experimental conditions the $\mathrm{C}_{2} \mathrm{H}_{2}^{+}$ fragments stay in the spectrometer's extraction region only during the first $0.37 \mu \mathrm{s}$, which is a small fraction of the whole time-of-flight. The similar observation is also valid for the $\mathrm{C}_{3} \mathrm{H}_{2} \mathrm{~N}^{+}$ fragment. According to general classification, fragment ions can be divided to three groups stable, metastable and unstable, where the decomposition rate for metastable ions lies between $10^{5}$ and $10^{6} s^{-1}$ [42]. So, stable ions will reach the detector without fragmentation, but instead of unstable ions their fragments will be detected. In terms of TOF measurements, the metastable ion is a fragment dissociating in flight from the extraction region to the field-free region of the mass spectrometer. In the case of acrylonitrile we have detected an abrupt increase of FWHM of time-of-flight peaks at around $15 \mathrm{eV}$ in binding energy scale (Table 2). We suggest to attribute the increase in KERs of $\mathrm{C}_{3} \mathrm{H}_{2} \mathrm{~N}^{+}$and $\mathrm{C}_{2} \mathrm{H}_{2}^{+}$fragments in spectator energy range of electron spectrum to the existence of metastable states, which cause the decomposition of corresponding fragments in flight from the extraction region to the field-free region of TOF analyzer. In both cases the KER values are below $100 \mathrm{meV}$, which indicates the absence of reverse activation barrier and simple bond cleavage dissociation reaction. The metastable state of protonated cyanoacetylene $(H C \equiv C H-C N)$ (or cyanovinylidene $\left(\mathrm{CH}_{2}=\dot{C}-C N\right)$ ) is probably the precursor for $C_{3} H N^{+}$ fragment, which appears to PEPICO map in this excess energy region. In the case of metastable fragmentation of $\mathrm{C}_{2} \mathrm{H}_{2}^{+}$, we expect the following reaction

$$
\mathrm{C}_{2} \mathrm{H}_{2}^{+} \rightarrow \mathrm{C}_{2} \mathrm{H}^{+}+\mathrm{H}
$$

This channel is most likely, if we consider the experimental and theoretical appearance energy of the $\mathrm{C}_{2} \mathrm{H}^{+}$fragment on PEPICO map. In other words, the metastable decay of the $\mathrm{C}_{2} \mathrm{H}_{2}^{+}$fragment appears if the internal excess energy exceeds the appearance energy of the $C_{2} H^{+}$. This assumption is also confirmed by earlier theoretical calculations and PEPICO experiment for $\mathrm{C}_{2} \mathrm{H}_{2}$ molecule $[44,45]$, where it was concluded that $\mathrm{C}_{2} \mathrm{H}_{2}^{+}$ions with internal energies $\geq 17.40 \mathrm{eV}$ decompose overwhelmingly into $\mathrm{C}_{2} \mathrm{H}^{+}$in a temporal scale $<12 \mu \mathrm{s}$. 
Table 2. KERs values of $\mathrm{C}_{2} \mathrm{H}_{2}^{+}$and $\mathrm{C}_{3} \mathrm{H}_{2} \mathrm{~N}^{+}$fragments determined from the data depicted in Figure 4. The error bars are given in brackets.

\begin{tabular}{|c|c|c|c|}
\hline Fragment & Binding energy (eV) & FWHM (ns) & KER (meV) \\
\hline$C_{2} H_{2}^{+}$ & $27 . .19$ & $46(3)$ & $60(12)$ \\
\hline$C_{2} H_{2}^{+}$ & $15 . .12$ & $20(1)$ & $10(2)$ \\
\hline$C_{3} H_{2} N^{+}$ & $20 . .15$ & $29(2)$ & $9(2)$ \\
\hline$C_{3} H_{2} N^{+}$ & $15 \ldots 12$ & $18(1)$ & $0(1)$ \\
\hline
\end{tabular}

\section{Conclusions}

The investigation of acrylonitrile dissociation following the core level excitations of cyano group atoms to the $\pi_{x y}^{*}(C \equiv N)$ orbital have been performed. The comparison of the electron-ion coincidence maps of the $N 1 s^{-1} \pi^{*}$ and $C 1 s^{-1} \pi^{*}$ Auger decay shows that in both cases the fragmentation channels are the same and the energy dependency on the initial site of core hole creation is very weak. On the other hand, the main features of these PEPICO maps strongly depend on the final cationic state reached by Auger decay of the core excited states. The fragmentation pattern clearly depends on the nature of resonant decay channel and/or the presence of excited electron. Also the migration of light $\mathrm{H}$ atoms during the excitation and after that plays an important role for the geometrical rearrangement of parent ion. Additionally, we have reported the existence of metastable states of acrylonitrile fragments, which depend on character of electronic decay channels and appear with the opening of the spectator Auger channels.

\section{Acknowledgments}

The experimental and theoretical work of this project was supported by the Academy of Finland, Nordforsk Infrastructure Network "Advanced spectroscopy using MAX-laboratory in Lund" and the EU "Transnational Access to Research Infrastructures" programm. Members of the electron spectroscopy group of the University of Oulu are acknowledged for their role in developing the PEPICO setup and sharing the equipment. We thank A. Caló and S. Urpelainen for their help during the experiment. Also the help of the staff of MAX-lab is greatly appreciated.

\section{References}

[1] S. Petrie, C.G. Freeman, M.J. McEwan, Mon. Not. R. astr. Soc. 257, 438, (1992).

[2] R.L. Hudson, M.H. Moore, Icarus 172, 466, (2004).

[3] H.K. Ervasti, K.J. Jobst, P.C. Burgers, P.J.A. Ruttnik, J.K. Terlouw, Int. J. Mass Spectrom. 262, 88, (2007).

[4] K.J. Jobst, S.A. Hasan, J.K. Terlouw, Chem. Phys. Lett. 450, 243, (2008).

[5] M.P. de Miranda, J.A. Beswick, P. Parent, C. Laffon, G. Tourillon, A.Cassuto, G. Nicolas, F.X. Gadea, J. Chem. Phys. 101, 5500, (1994).

[6] I. Wilhelmy, C. Laffon, H.-U. Ehrke, W. Wurth, J. Phys. Chem. 99, 8496, (1995).

[7] Ph. Parent, C. Laffon, G. Tourillon, A. Cassuto, J. Phys. Chem. 99, 5058, (1995).

[8] M.-J. Hubin-Franskin, H. Aouni, D.Duflot, F. Motte-Tollet, C. Hannay, L.F. Ferreira, G. Tourillon, J. Chem. Phys. 106, 35, (1997).

[9] F. Motte-Tollet, D. Messina, M.-J. Hubin-Franskin, J. Chem. Phys. 103, 80, (1995). 
[10] S. Eden, P. Limão-Vieira, P. Kendall, N.J. Mason, S.V. Hoffmann, S.M. Spyrou, Eur. Phys. J. D 26, 201, (2003).

[11] A. Naves de Brito, S. Svensson, H. Ågren, J. Delhalle, J. Electron Spectroscopy Relat. Phenomena 63, 239, (1993).

[12] V.M. Geskin, R. Lazzaroni, M. Mertens, R. Jérôme, J.L. Brédas, J. Chem. Phys. 105, 3278, (1996).

[13] X. Crispin, C. Bureau, V.M. Geskin, R. Lazzaroni, W.R. Salaneck, J.L. Brédas, J. Chem. Phys. 111, 3237, (1999).

[14] F. Bournel, J.-J. Gallet, S. Kubsky, G. Dufour, F. Rochet, M. Simeoni, F. Sirotti, Surf. Sci. 513, 37, (2002).

[15] M. Simeoni, L. Lozzi, S. Santucci, Surf. Sci. 540, 55, (2003).

[16] S. Rangan, S. Kubsky, J.-J. Gallet, F. Bournel, K. Le Guen, G. Dufour, F. Rochet, R. Funke, M. Kneppe, G. Piaszenski, U. Köhler, F. Sirotti, Physical Review B 71, 125320, (2005).

[17] J.-J. Gallet, F. Bournel, S. Kubsky, G. Dufour, F. Rochet, F. Sirotti, J. Electron Spectroscopy Relat. Phenomena 122, 285 (2002).

[18] K. Głuch, J. Cytawa, L Michalak, Int. J. Mass Spectrom. 278, 10, (2008).

[19] K. Głuch, E. Szot, A. Gruszecka, M. Szymańska-Chargot, J. Cytawa, L Michalak, Vacuum 83, S20, (2009).

[20] A. Fahr, A. H. Laufer, J. Phys. Chem. 96, 4217, (1992).

[21] C. A. Bird, D. J. Donaldson, Chem. Phys. Lett. 249, 40, (1996).

[22] S. W. North, G. E. Hall, Chem. Phys. Lett. 263, 148, (1996).

[23] D. A. Blank, A. G. Suits, Y. T. Lee, S. W. North, G. E. Hall, J. Chem. Phys. 108, 5784, (1998).

[24] M. J. Wilhelm, M. Nikow, L. Letendre, H.-L. Dai, J. Chem. Phys. 130, 044307, (2009).

[25] A. Derecskei-Kovacs, S. W. North, J. Chem. Phys. 110, 2862, (1999).

[26] W.-N. Du, C. Luo, Z.-S. Li, J. Chem. Phys. 129, 174309, (2008).

[27] E. Itälä, E. Kukk, D. T. Ha, S. Granroth, A. Caló, L. Partanen, H. Aksela, S. Aksela, J. Chem. Phys. 131, 114314, (2009).

[28] J. H. D. Eland, Int. J. Mass Spectrom. Ion Phys. 8, 143, (1972).

[29] C. J. Danby, J. H. D. Eland, Int. J. Mass Spectrom. Ion Phys. 8, 153, (1972).

[30] Frontiers of Coincidence Experiments, in J. Electron Spectroscopy Relat. Phenomena, edited by K. Ueda 141, (2004).

[31] E. Kukk, R. Sankari, M. Huttula, A. Sankari, H. Aksela, S. Aksela, J. Electron Spectroscopy Relat. Phenomena 155, $141(2007)$.

[32] M. Huttula, S. Heinäsmäki, H. Aksela, E. Kukk, and S. Aksela, J. Electron Spectroscopy Relat. Phenomena 156-158, 270 (2007).

[33] E. Kukk, Spectral Analysis by Curve Fitting Macro Package /SPANCF/ 2000 http://www.physics.utu.fi/en/research/material science/Fitting.html

[34] M. Bässler, A. Ausmees, M. Jurvansuu, R. Feifer, J. -O. Forsell, P. de Tarso Fonseca, A. Kivimäki, S. Sundin, S. L. Sorensen, R. Nyholm, O. Björneholm, S. Aksela, and S. Svensson, Nucl. Instrum. Methods in Phys. Res A 469, 382 (2001).

[35] P. A. Mullen, M. K. Orloff, Theoret. chim. Acta (berl.) 23, 278 (1971).

[36] K. Kimura, S. Katsumata, Y. Achiba, T. Yamasaki, S. Itawa, Handbook of He I Photoelectron Spectra of Fundamental Organic Molecules; Japan Scientific Societies Press: Tokyo, 1981.

[37] V. Ilakovac, S. Carniato, J.-J. Gallet, E. Kukk, D. Horvatic and A. Ilakovac, Physical Review A 77, 012516 (2008).

[38] R. Krishnan, J. S. Binkley, R. Seeger, J. A. Pople J. Chem. Phys. 72, 650 (1980).

[39] M. W. Schmidt, K. K. Baldridge, J. A. Boatz, S.T.Elbert, M. S. Gordon, J. J. Jensen, S. Koseki, N. Matsunaga, K. A. Nguyen, S. Su, T. L. Windus, M. Dupuis, J. A. Montgomery, J. Comput. Chem. 14, 1347 (1993). GAMESS (http://www.msg.chem.iastate.edu/gamess/)

[40] P. Culot, G. Dive, V. H. Nguyen and J. M. Ghuysen, Theoret. Chimica Acta 82, 189 (1992).

[41] J. Laskin, C. Lifshitz, J. Mass Spectrom. 36, 459, (2001).

[42] J.H. Gross, Mass Spectrometry. A Textbook (Springer-Verlag, Berlin Heidelberg, 2004)

[43] T. Osipov, C. L. Cocke, M. H. Prior, A. Landers, Th. Weber, O. Jagutzki, L. Schmidt, H. Schmidt-Böcking, R. Dörner, Phys. Rev. Lett. 90, 233002 (2003).

[44] W. P. Kraemer, W. Koch, Chem. Phys. Lett. 212, 631 (1993). 
[45] K. Norwood, C. Y. Ng, J. Chem. Phys. 91, 2898 (1989). 